\title{
Construction of System to Support Intelligent Coffee Shop using IoT
}

\author{
Byeongtae Ahn
}

\begin{abstract}
Recently, the development of smart devices linked to the network and the diffusion of the Internet have increased the user convenience. This system maximizes the efficiency by combining the uncomfortable points in the existing cafe with the internet technology. In particular, smartphone orders can be ordered including kiosk orders, and beacon-based users can be automatically recognized. And we have developed a system that enables users to create customized orders by grasping user location information using geofences. Finally, this paper also provides weather, temperature, time and user-based recommendation services based on Big Data. In particular, it supports intelligent decision making system through big data analysis. Therefore, Real-time reservations and orders using smart phones are supported from outside. In this paper, a Smart Café system is constructed with smart devices and Internet devices being shared and cooperatively controlled, thereby reducing labor costs and enhancing user convenience.
\end{abstract}

Data

\section{INTRODUCTION}

As various types of smart devices including smart phones become popular, various products equipped with smart functions are being released. The ultimate goal of an intelligent application running on a smart phone is to judge and provide appropriate services without requiring the user to have the information necessary to execute the service. To develop an intelligent application that meets these needs, context for the user and the user's environment is needed. Context is used to get context information of an entity [1]. Sensors mounted on smartphones, which are currently popular, provide important source data for acquiring user behavior and surrounding information. The convergence of Internet technology and smart device technology enables smart device services, which were provided by existing server-client methods, to be replaced by Internet based services in terms of cost and resource efficiency[2].

Therefore, in this paper, a smart cafe system based on the Internet of things has been constructed for efficiency improvement and effectiveness in a single café, which is a typical startup company of small business owners [3]. This system is a smart cafe system that combines the inconveniences and ineffective functions of existing coffee shops with IoT technology and smart functions. In Section 2 of this paper, related works are introduced. In Section 3, the system is designed. In Section 4, a system based on system design is constructed. Section 5 presents conclusions and future work.

\section{RELATED WORK}

Object Internet refers to a network of objects and spaces that forms intelligent relationships such as sensing, networking, and information processing in a cooperative manner without human intervention for the distributed environmental elements of human, object, and service [4]. In addition, it refers to intelligent networking technology and environment in which surrounding objects are connected to wired / wireless networks to organically collect and share information [5]. The Internet has been used in a variety of fields and includes many technical elements. In 1999, Kevin Ashton said, "By installing Radio Frequency Identification (RFID) and other sensors on objects in everyday life, a huge Internet of things will be built," the term Internet of Things (IoT) For the first time. Since then, in 2005, the ITU (International Telecommunication Union) 's Internet related reports 1) The Internet became more known by establishing the concept of the Internet and presenting related technologies and development directions.

Objects The Internet is a concept that collectively refers to technologies and services in which information such as people, objects, and data are all connected to the Internet and generated, collected, shared, and utilized. In particular, the Internet of things is a technology that allows objects to be connected to the Internet without human intervention, communicating and exchanging data without human recognition. The Internet of Things The past has evolved from ubiquitous concepts to P2P (Person to Person), P2M (Person to Machine) and Machine to Machine (M2M), and extends communication objects to all objects and the Internet.

The core technology of the Internet of things is sensing technology, wired and wireless network technology, and platform technology. Sensing technology recognizes information such as temperature, humidity, heat, illumination, gas, motion, image, radar, etc., and transfers it to the digital world. Wireless and wired network technology is not limited to time and place but is a technology that information Internet devices connect to the transmission network to transmit and receive information. It is used for RFID, NFC, Wi-Fi, ZigBee, Global System for Mobile communications In addition to short-distance communication, network technology that enables long-distance communication such as $3 \mathrm{G}$ and LTE is utilized in the Internet of things. 
Platform technology is a technology to connect and manage heterogeneous devices connected to the Internet without collision.

The Internet technology of things is being used in all industries such as city / safety, health / medical, energy, manufacturing, automobile, home. Among them, It is actively applied in natural disaster monitoring such as chemical water management, forest fire monitoring and slope management, hazardous material detection / alarm service, prevention of childbearing, and child safety service.

Object The networking technology of the Internet is a wired / wireless networking function that can connect distributed environmental elements such as people, objects, and services to each other, and is developing around wireless networks. IP-based protocol technology for exchanging information based on Internet connection such as WIFI, 3G / 4G / LTE etc. is used for connection between devices, and Bluetooth (Bluetooth), which is used for communication between devices / When non-IP protocols such as Zigbee, Zwave, and RFID are used, information can be shared with the Internet or other devices through a sink node [6]. In a home network environment for a smart home service, devices are connected through a WIFI mainly around a wireless access point (AP). Recently, various products such as SmartThings, Withings, and Philips have launched smart bulbs and sensors that can be used in a home environment [7]. These smart sensors and actuators mainly use ZigBee / ZWave to exchange information between devices, and they send and receive control and status messages through a sink node called Hub / Bridge and connected to the Internet [8] [9].

\section{DESIGN OF INTELLIGENT COFFEE SHOP MANAGEMENT SYSTEM}

The intelligent coffee shop management system proposed in this paper is designed to operate effectively by combining IoT technology with inconveniences when it is seen from the perspective of customers and operators of existing coffee shops. First, the order service function is provided using kiosk and smartphone app. Customers can enter the coffee shop and order directly from the kiosk and make payments. And when you make a payment at the same time using the smartphone app from outside the coffee shop, you can receive the order without wait by providing the waiting time information. In particular, by using beacons in a kiosk, it is possible to easily recognize the user, thereby enabling efficient ordering. Second, it provides weather, temperature, sales volume, order history, and advertisement based recommendation service in real time through big data analysis. Big Data Analysis receives real-time information from Meteorological Agency and Coffee Shop server and recommends customized customer order.

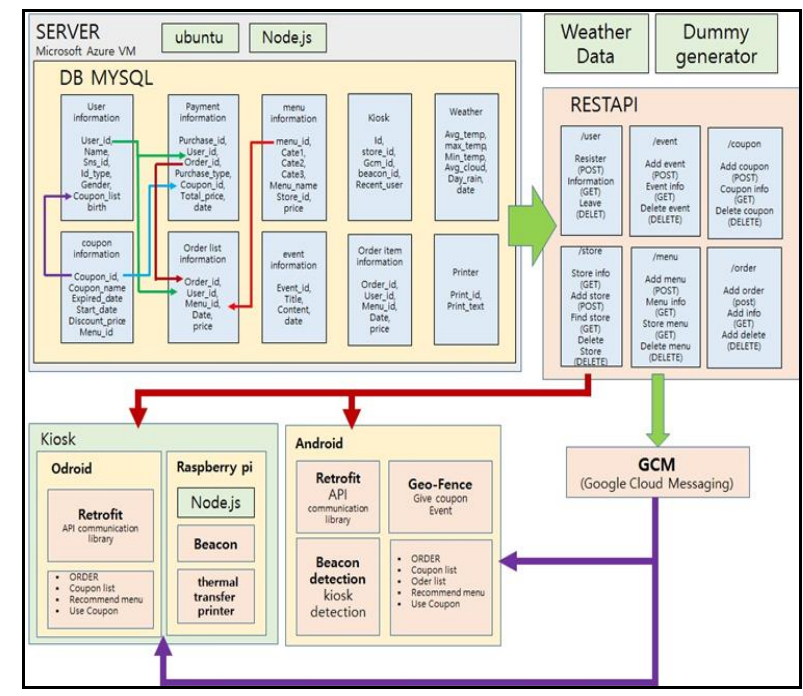

Fig. 1. Structure of System

Third, a discount coupon is provided using the Geo-Fence when the coffee shop is located at a distance of 100 meters. Geo-Fence has made it possible to receive information only from customers who have signed up. Fourth, it solved the hassle of waiting in line for online ordering, payment, and customer attraction. Fig. 1 shows the overall system configuration of the intelligent coffee shop. The server uses MYSQL DB and Node. Js and uses Google's cloud message to provide data to the smartphone in real time.

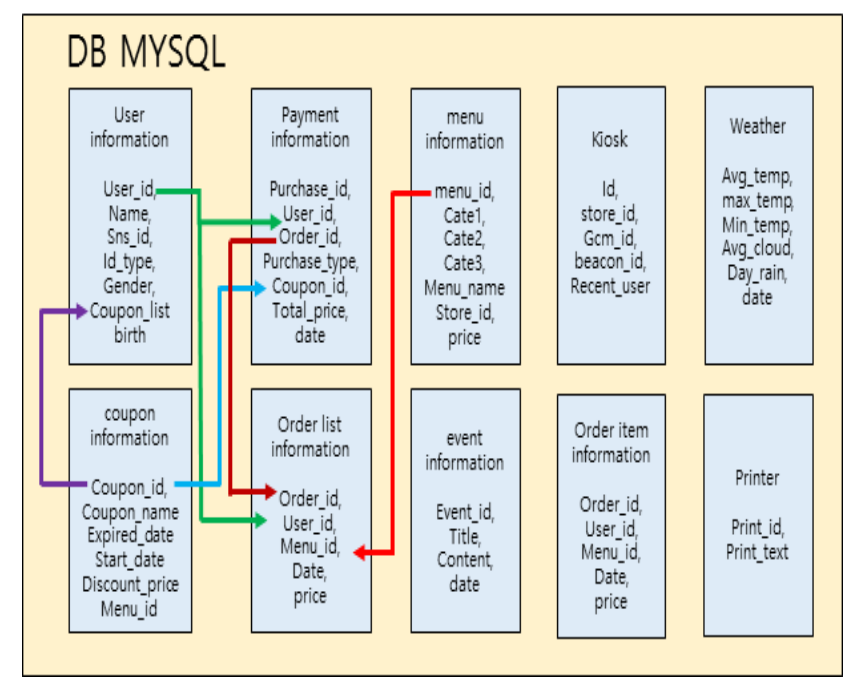

Fig. 2. Structure of DB

Fig. 2 shows the database structure of the smart cafe system server. It is made up of 10 tables, and it has user information and output information table. 


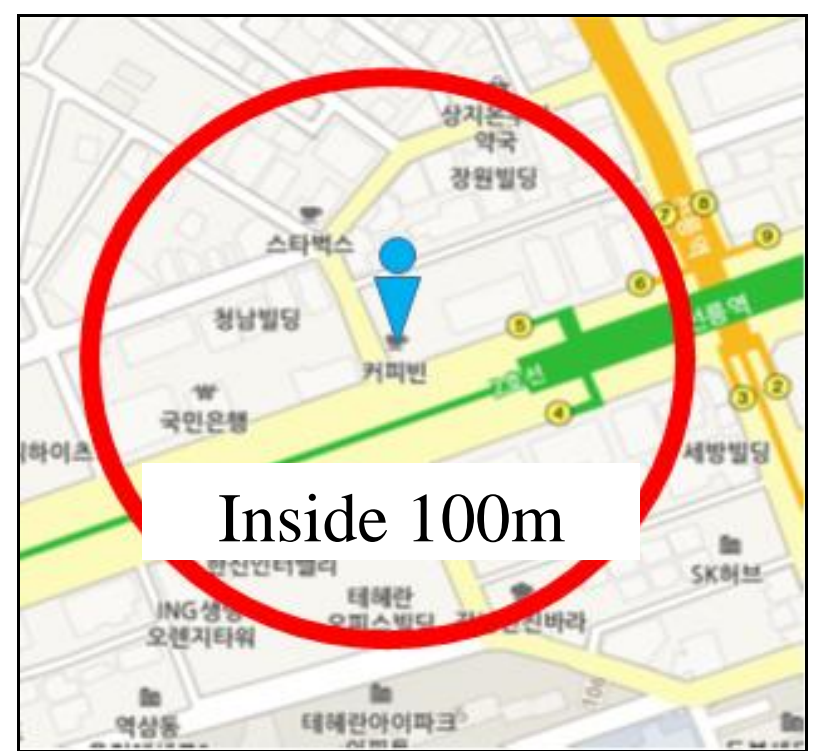

Fig. 3. Event using Geo-Fence

Fig. 3 shows an event using Geofence[11]. If customers have a radius of 100 meters based on the branch, they attract customers by offering discount coupons and service coupons in real time. The customer's location information was approved by the subscription as an option, and customer situation information within a 100-meter radius was grasped. One minute later, the customer's location information was grasped again and the coupon was provided as soon as it was close to the location.

\section{IMPLEMENTATION OF INTELLIGENT COFFEE SHOP MANAGEMENT SYSTEM}

In this paper, we provide weather, temperature, sales volume, order history, and advertisement based recommendation service in real time through order service system using kiosk and smartphone app and big data analysis. And, when approaching a cafe location radius of $100 \mathrm{~m}$, we provided cafe event coupon using Geo-Fence free of charge.

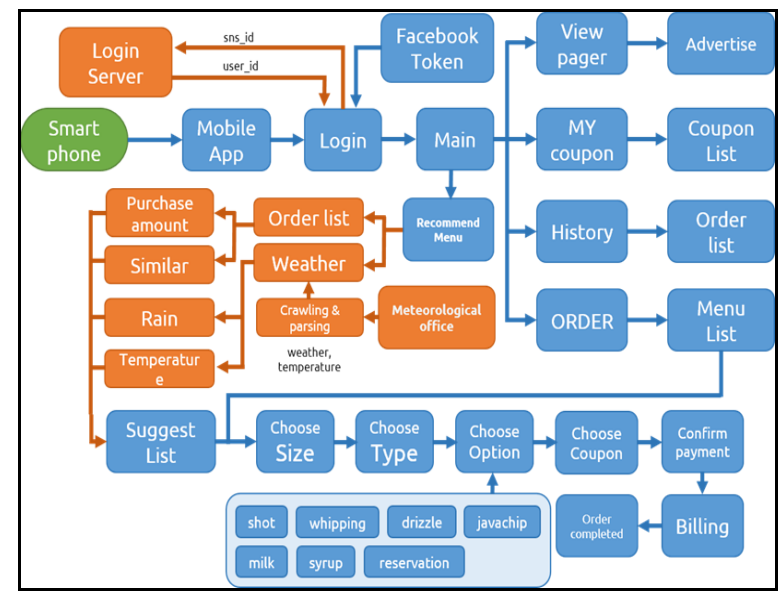

Fig. 4. Interface of smartphone

Fig. 4 shows the initial screen of smart cafe app running on smartphone. When the app is installed on the smartphone and connected, the server confirms the member information and supports connection. When the member information is confirmed, the smart cafe main screen appears and provides weather list information, recommendation menu including order list. The coupon list provides the current available coupon information in real time and the customer can place the order at the optimized price.

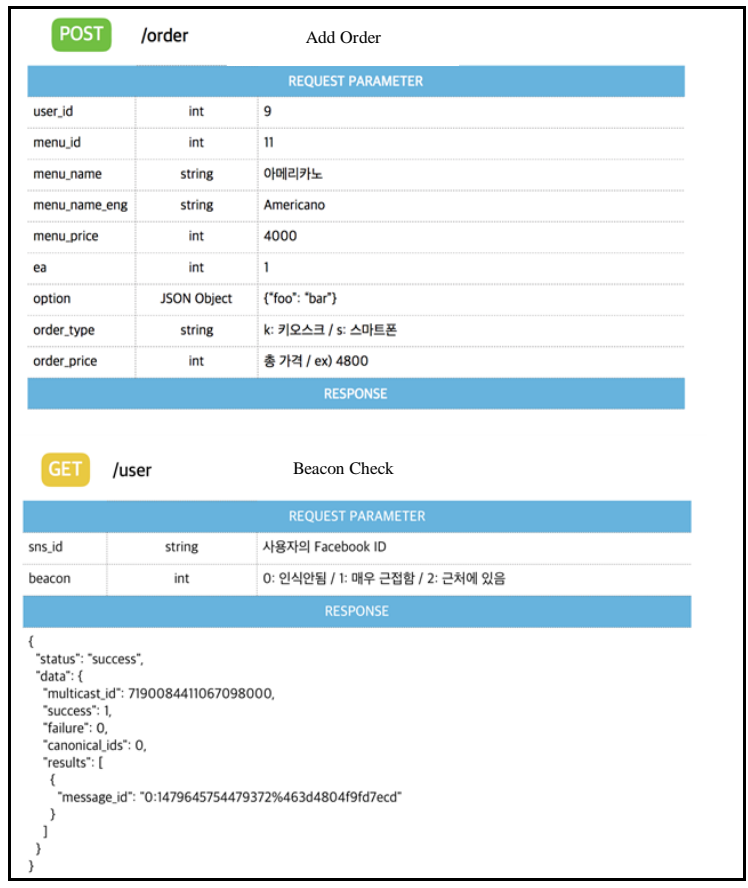

Fig. 5. Structure of Order/Becon Table

Fig. 5 shows the configuration diagram of the order information and beacon signal information stored in the table. The signal information transmitted by the smartphone is recognized as a beacon and stored in real time. It is the source of the format in which signals are actually received and stored.

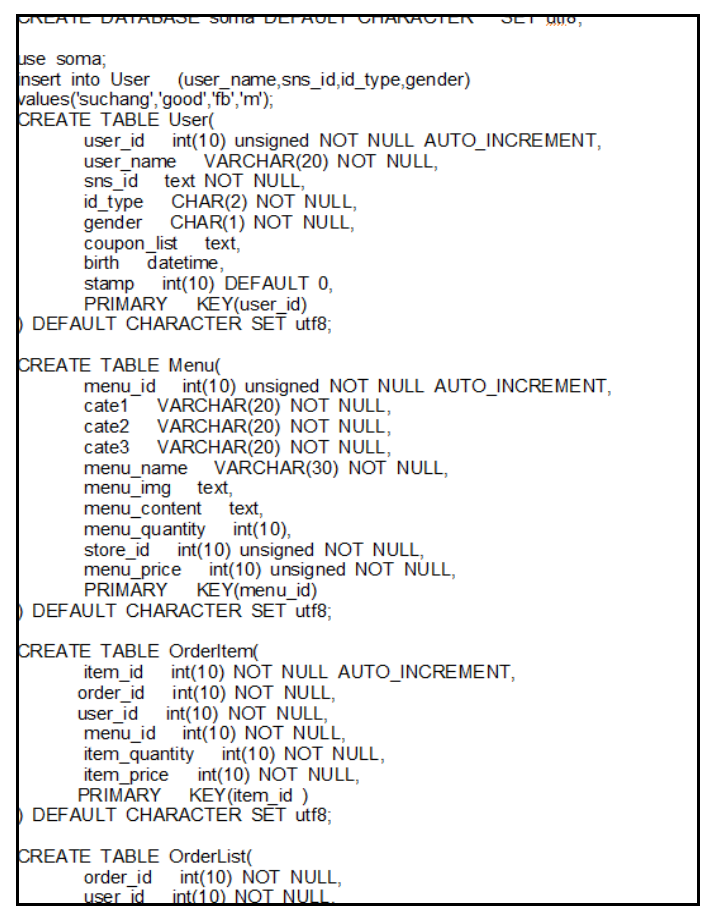

Fig. 6. Source of DB 


\section{Construction of System to Support Intelligent Coffee Shop using IoT}

Fig. 6 builds an order-related table based on the database design provided in Figure 2 of Chapter 3, Design. In the order table, an order price item is set up including a user identification item. The table below is a table for storing information in beacon recognition. Figure 6 shows the database source we built in Chapter 3. Each item consists of a user table, a menu table, an order item table, and an order list table.

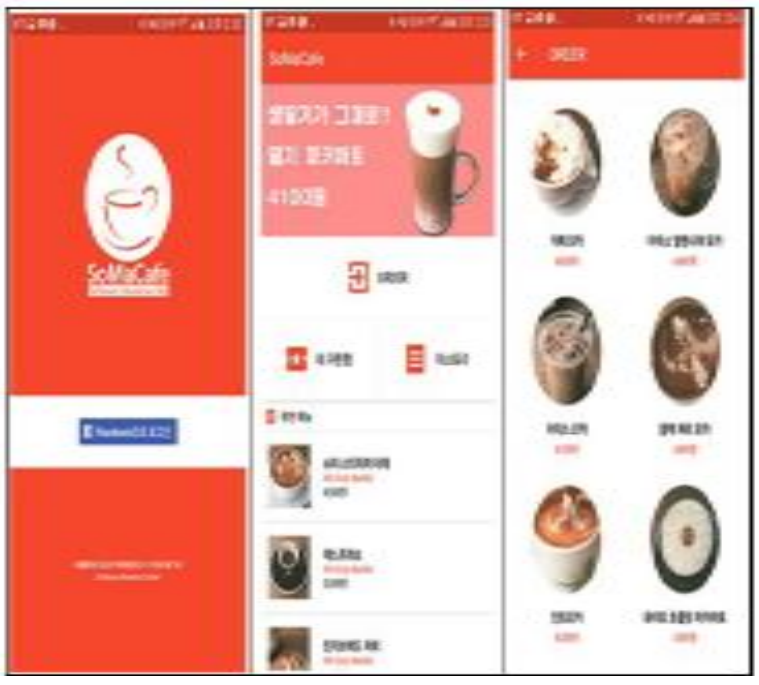

Fig. 7. Interface of smartphone

Fig. 7 shows an initial screen where a user places an order with a login on a smartphone. The first picture on the left is the initial screen for signing up and logging in to the cafe, and the middle picture shows the coupon box with the order item price. The coupon box uses Geo-Fence to guide the customer to the store by providing a coupon when the customer arrives within a radius of 100 meters from the store. The figure on the right lists prices and pictures by item.

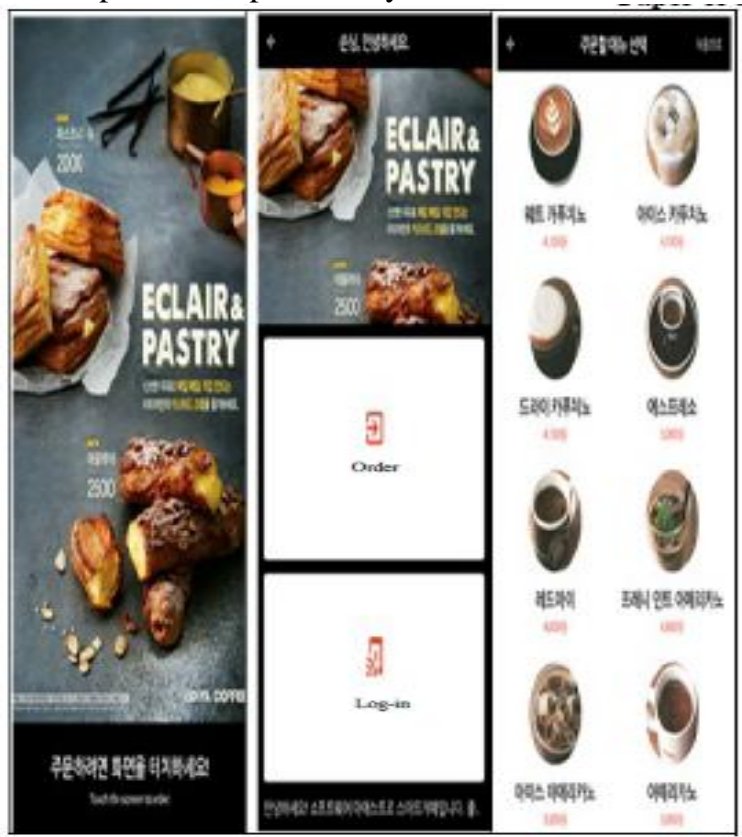

Fig. 8. Interface of kiosk

Fig. 8 shows the initial screen of the kiosk. The left figure is the initial screen of the kiosk. When the customer is in front of the screen, the customer information is displayed on the screen by beacon recognition. Customers can use the kiosk to place orders without lining the store. If the customer is not in front of the kiosk, he or she will promote the new menu of the store. The middle picture is the initial screen just before ordering after customer's member certification. If you click "Order", the order list appears as shown on the left, and the customer can place orders directly.

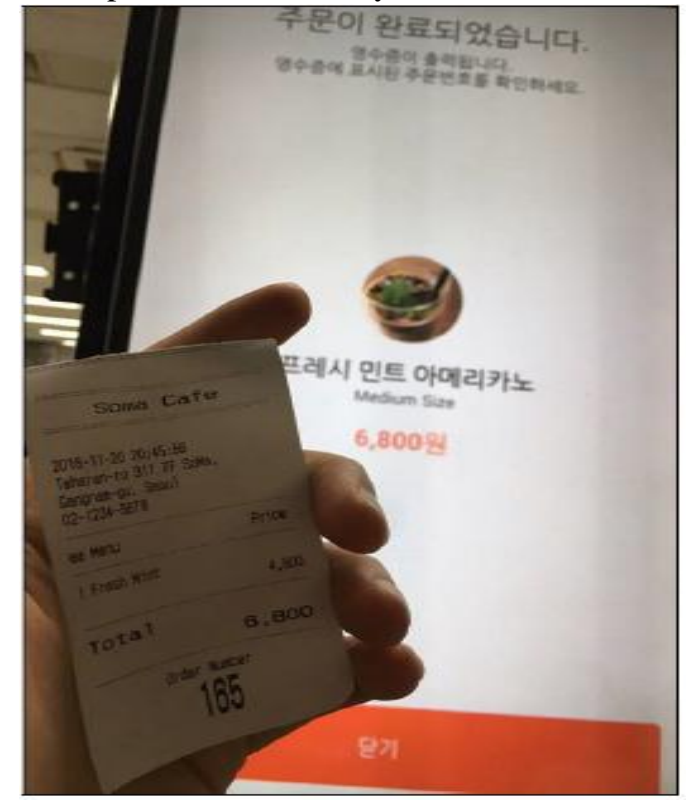

Fig. 9. Output of order information

Fig. 9 shows the receipt printed on the electronic print under the kiosk when you make a payment at the kiosk. Order information is provided on the receipt and customers can find the order when the order number of the store is available.

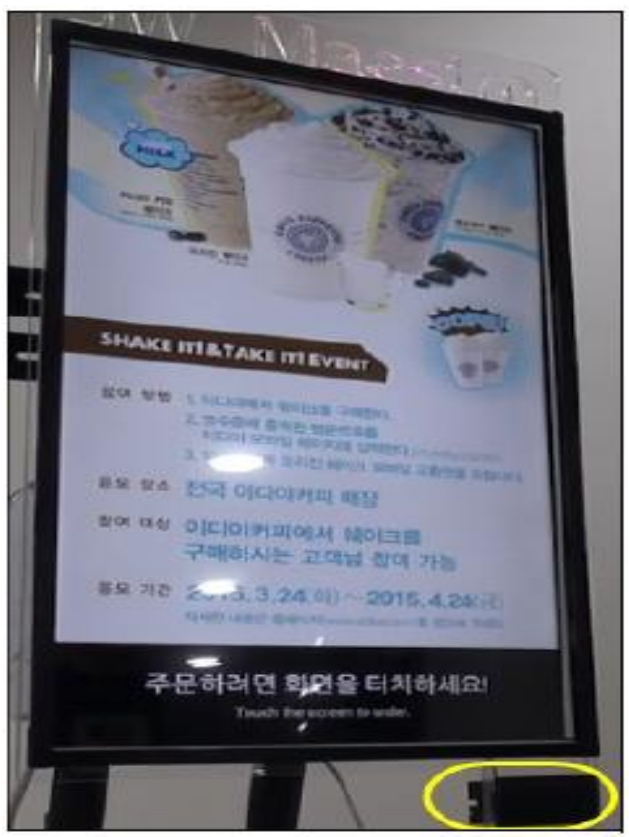

Fig. 10. Kiosk equipment

Fig. 10 shows the kiosk equipment. What is in the circle in Figure 8 is the printer that prints the receipt. This equipment is to make the sensor operate by putting patellar on the existing LCD monitor. 


\section{CONCLUSION}

In this paper, we constructed a service that provides weather, temperature, sales volume, order history, and advertisement based recommendation service in real time through order service system using kiosk and smartphone app and big data analysis. And, when approaching a cafe location radius of $100 \mathrm{~m}$, we provided cafe event coupons free of charge using geofence. By providing offline online ordering, payment, and customer attraction online in real time, we improved indirect costs and efficiency. As a future work, we will implement the system so that it will work on iOS as it will only work on Android base.

\section{REFERENCES}

1. Agah Tugrul Korucu, Ayse Alkan. Procedia-Social and Behavioral Sciences. 2017 jun;15:1925-1930.(Differences between m-learning (mobile learning) and e-learning, basic terminology and usage of m-learning in education)

2. Leonardo A. Amaral, Fabiano P. Hessel, Eduardo A. Bezerra, Jerônimo C. Corrêa, Oliver B. Longhi, Thiago F. O. Dias. Journal of Network and Computer Applications. 2017 May;34(3):972- 979(eCloudRFID - A mobile software framework architecture for pervasive RFID-based applications)

3. Tsung-Han Chang, Shu-Chen Hsu, Tien-Chin Wang. Applied Mathematical Modeling. 2016 Mar;37(5):2605-2622(A proposed model for measuring the aggregative risk degree of implementing an RFID digital campus system with the consistent fuzzy preference relations)

4. Chih-Ming Chen. Expert Systems with Applications. 2010 Sep;37(9):6651-6662. (Intelligent location-based mobile news service system with automatic news summarization)

5. Agah Tugrul Korucu, Ayse Alkan. Procedia-Social and Behavioral Sciences. 2011 Dec;15:1925-1930.(Differences between m-learning (mobile learning) and e-learning, basic terminology and usage of m-learning in education)

6. M. C. Chan, Y. Ding, K. H. Chai. Management of Innovation and Technology. 2008 Sep; 934-939. (The collective effects of product and service quality on customer satisfaction-An empirical study on iPod and iTunes)

7. Sasan adibi. Telematics and Informatics. 2017 Nov;27(4): 377-393. (A remote interactive non-repudiation multimedia-based m-learning system)

8. H. S. Lee, S. H. Lee. Journal of digital Convergence. 2017 May;14(5):1-10(Impact on Internalization of Management Strategy in Public Organization)

9. D. S. Lee. Journal of the Korea Convergence Society. 2017 Feb;8(2):15-20. (Design of Compact Data Integration and Convergence Device Using Esp8266 Module)

10. J. C. Lee. Journal of the Korea convergence Society. 2017 Feb;8(2):27-33. (A Classification Algorithm using Extended Representation)

11. J. Kim. Journal of Korean Institute of Information Technology. 2015 Oct;13(10):31-36. (Computer Vision Based Lamp Control System by using Raspberry-Pi)

\section{AUTHORS PROFILE}

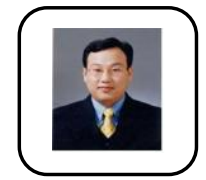

Byeong-Tae Ahn is a Faculty of Division of Liberal Arts at Anyang University, Korea. His research interests include: Image Processing, Video Analysis, IoT, BlockChain, Multimedia Database and MPEG-7. His address is: 37-22, Samduck Minahn-gu Anyang-City Gyeonggi-do, 430-714 South Korea. His phone number is +82-31-463-1204 and the email address is ahnbt@anyang.ac.kr. 